\title{
PHYSICAL PROPERTIES OF RAPIDLY DECAYING AFTERGLOWS
}

\author{
M. De Pasquale ${ }^{1}$, S. Schulze ${ }^{2}$, D.A. Kann ${ }^{3}$, S. Oates ${ }^{4}$ and B. Zhang ${ }^{1}$
}

\begin{abstract}
We analyze a sample of Swift X-ray and optical GRB afterglows which were chosen so as to reduce selection effects against the weakest events. We tentatively identify important differences in GRBs that show a fast decay with respect to the average GRB, in particular a tendency for the more energetic bursts to have a steeper afterglow decline. An explanation to this finding could be bursts in a wind environment are on average more energetic than bursts occurring in a constant density medium.
\end{abstract}

\section{Introduction}

The Swift mission (Gehrels et al. 2004) has ushered in an era of intense study of GRB afterglows. The high detection rate, 100 GRBs/year, the prompt observations in X-ray and optical bands that begin $\sim 100 \mathrm{~s}$ after the trigger, and the rapid dissemination of the coordinates to ground facilities have enabled observers to build large samples of multi-wavelength GRB afterglow light curves, that cover evolution from GRB onset to days or weeks later. We can therefore model the temporal and spectral behaviour in great detail and draw conclusions on the physical parameters of the bursts. In this work, we build on the analysis of Schulze et al. (2011), trying to understand whether there is any possible correlation between the decay slope of the afterglows and the energetics of the GRB.

\section{Initial sample and its properties}

Schulze et al. (2011) examined the complete dataset of Swift bursts occurring between launch and September 2009. They selected events that obeyed the following

\footnotetext{
${ }^{1}$ Department of Physics \& Astronomy, University of Nevada, Las Vegas, USA

${ }^{2}$ Pontificia Universidad Católica de Chile, Chile

3 Thüringer Landessternwarte Tautenburg and Max-Planck-Institut für extraterrestrische Physik, Germany

${ }^{4}$ Mullard Space Science Laboratory, University College London, UK
} 

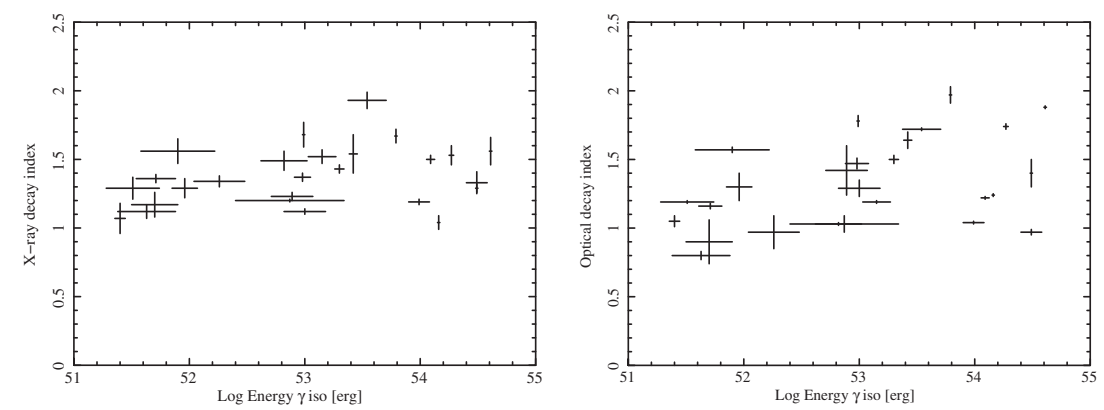

Fig. 1. Left: plot of X-ray decay slope $\alpha_{X}$ against the energy released in $\gamma$-rays at the prompt phase in the sample of Schulze et al. (2011). Right: same as left panel, but displaying $\alpha_{o p t}$ rather than $\alpha_{X}$.

criteria: i) known redshift (and thus known energetics); ii) X-ray and optical afterglows are not dominated by flares or bad data sampling iii) both the X-ray and optical afterglows satisfy the forward shock closure relations (Sari et al. 1998; Sari et al. 1999; Chevalier \& Li 2000) in the adiabatic case, i.e., the slow decline phase (Nousek et al. 2006) is not included in the study. For most events in Schulze et al. (2011), it is possible to distinguish between a circumburst medium with constant density profile (Insterstellar medium like, ISM) and a stellar wind profile (wind). In the left and right panels of Figure 1, we show the decay slopes of the X-ray and optical bands, $\alpha_{X}$ and $\alpha_{o p t}$, versus the energy released in $\gamma$-rays during the prompt phase assuming isotropy, $E_{\gamma, i s o}$, for this sample. The probability of a correlation, assessed given the value of Pearson correlation coefficient between $\alpha_{X}$ and $E_{\gamma, \text { iso }}$, is found to be less than $90 \%$, thus it is not statistically significant. However, we find that a trend between $\alpha_{o p t}$ and $E_{\gamma, i s o}$ is more likely: the probability of a correlation, evaluated as above, is $97 \%$.

\section{Correlation tests}

Motivated by these findings, we have used the same criteria as Schulze et al. (2011) to extend the previous sample to include bursts which occurred in 2010 and 2011. In the left and right panels of Figure 2, we show the decay indices against $E_{\gamma, \text { iso }}$ for this revised sample. The probability of a correlation between $\alpha_{X}$ and $E_{\gamma, \text { iso }}$ is now $99 \%$, while between $\alpha_{\text {opt }}$ and $E_{\gamma, \text { iso }}$ it is $99.86 \%$. By enlarging the sample, we find the probability for correlation has increased.

An immediate possibility to explain this correlation is that the most energetic bursts are already in the jet-break regime. Bursts with higher $E_{\gamma, \text { iso }}$ may have an earlier break, which is attributed to a collimated outflow. However, in the sample at hand, only $\sim 20 \%$ of bursts have temporal indices consistent with a jet regime. If we remove such events, there is still a residual marginal trend, since the probability of a correlation between the decay slope and the energetics becomes $97.5 \%$. While 

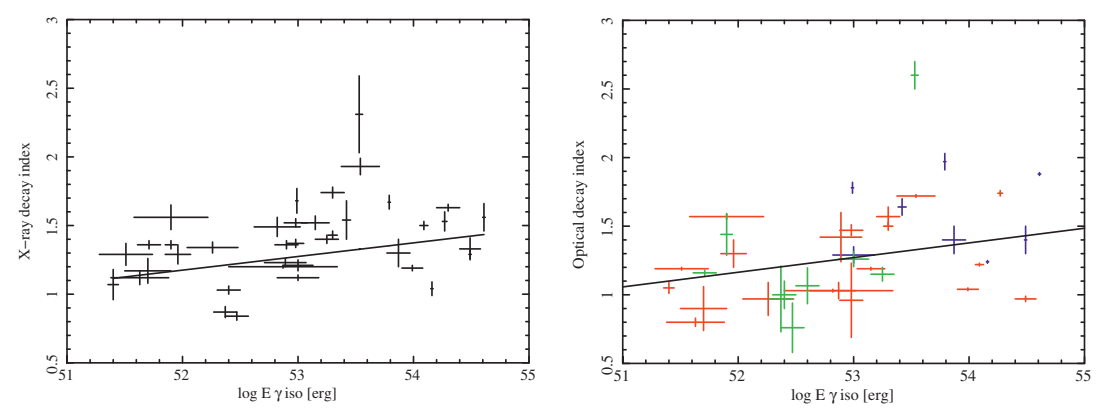

Fig. 2. Left: plot of the X-ray decay slope $\alpha_{X}$ against the energy released in $\gamma$-rays during the prompt phase in our updated sample. The line represents a linear fit of the data, in which only 1-D errors are taken into account. Right: same as left panel, but displaying $\alpha_{\text {opt }}$ rather than $\alpha_{X}$. Blue, red and green points represent GRBs in wind environments, those in an ISM, and events for which the environment cannot be distinguished, respectively.

the statistical strength of this residual correlation is weak, we may speculate what could cause it, if it turns out to be real. The optical band is usually below the cooling frequency $\nu_{c}$; the forward-shock model predicts that the slope, under such a condition, is steeper in a wind than in an ISM environment, all other parameters being equal. Thus, bursts with steeper decaying optical slopes in our sample may be those in a wind environment, which combined with the correlation between $\alpha_{o p t}$ and $E_{\gamma, i s o}$ would imply that bursts in a wind medium are, on average, also more energetic. This scenario could also explain why the X-ray decay slope is not strongly correlated with $E_{\gamma, i s o}$ : contrary to the optical band, the X-ray band is usually above $\nu_{c}$, and the decay slope, under such a condition, is not affected by the density profile of the medium. An examination of Table 3 in Schulze et al. (2011) may corroborate the scenario in which wind bursts are on average more energetic than those in an ISM. In this table, bursts in constant density medium span 4 decades in $E_{\gamma, i s o}$, between $\sim 10^{51}$ to a few $10^{54} \mathrm{erg}$, while bursts in wind medium are all above $10^{53} \mathrm{erg}$. Looking at this in another way, this shows that $20 \%$ of burst in an ISM have $E_{\gamma, i s o}>10^{54} \mathrm{erg}$, while $50 \%$ of bursts in wind are above this threshold. In the left panel of Figure 3, we plot a histogram of $E_{\gamma, \text { iso }}$ of the bursts of our sample, distinguishing between those consistent with ISM and Wind medium. While these two sub-samples overlap, it seems that wind bursts are on average more energetic. A Kolmogorov-Smirnov test indicates that the two distributions may not be drawn from the same population $(\mathrm{P}=1.8 \%)$. Finally, if we remove bursts in wind medium (blue points in right panel of Fig. 4), any hint of a correlation between optical decay slope and energetics basically disappears, since the probability decreases to $\sim 80 \%$. All these hints point toward a scenario in which bursts in a wind medium are, on average, more energetic than those in an ISM, although they unquestionably do not prove it. 

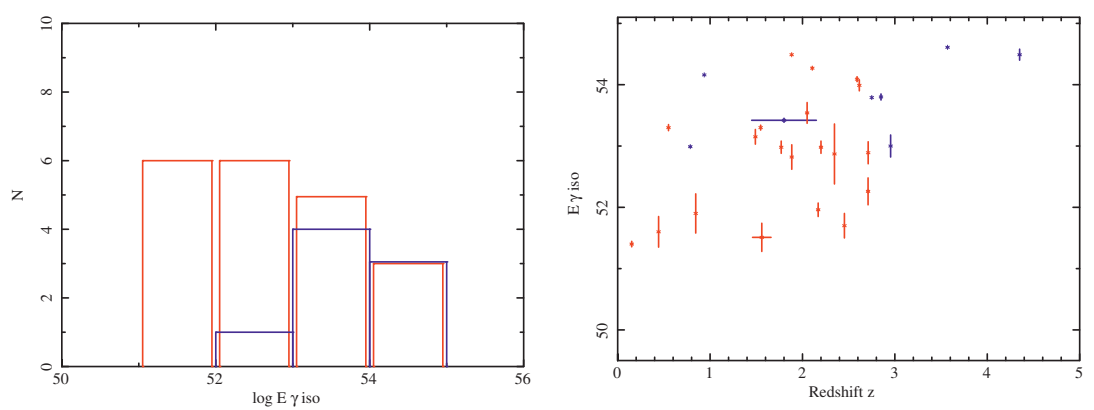

Fig. 3. Left: histogram with bursts found to be in a wind environment (blue) and an ISM (red). Right: the energy released in $\gamma$-rays versus redshift, bursts found to be in an ISM are red and wind are blue.

\section{Possible bias}

The speculations above, however, may be affected by the following bias. Due to the steeper decays, bursts in wind media generally need brighter afterglows to satisfy the criteria of the sample, and brighter afterglows generally imply higher $E_{\gamma, \text { iso }}$ as well. However, we find that this bias might not be pronounced. In the right panel of Figure 3 we plot $E_{\gamma, i s o} v s$. redshift. If the aforementioned bias was strong, one would expect to see a concentration of less energetic bursts at low redshift. However, this does not appear to be true.

We caution also that we are still within the regime of low-number statistics, for several bursts the density profile of the medium could not be ascertained, and we could only study those events for which data were published. One will need a larger and more complete sample to ascertain conclusively whether bursts in wind have larger $E_{\gamma, \text { iso }}$ than explosions in an ISM or not.

\section{Conclusions}

Within a sample of Swift bursts obeying the forward shock model, we find a strong indication for a correlation between $E_{\gamma, \text { iso }}$ and optical decay index. A correlation between $E_{\gamma, \text { iso }}$ and X-ray band decay index is less significant.

These correlations can be explained by the fact that a few energetic events are already in the post jet-break regime at early epoch. However, if we take these events out, we still have an indication for a correlation between $\alpha_{\text {opt }}$ and $E_{\gamma, \text { iso }}$. This correlation, if turns out to be real, may imply that bursts in stellar wind environment are on average more energetic that those in an ISM. The probability that distributions of $E_{\gamma, \text { iso }}$ of wind and ISM bursts are drawn from the same population is $1.8 \%$. Observational biases which would favour brighter wind bursts need to be carefully taken into account, and we shall need a larger sample to 
establish whether the progenitors of the most energetic bursts are also more likely to shape the environment around them with their stellar winds.

\section{References}

Chevalier, R.A., \& Li, Z.Y., 2000, ApJ, 536, 195

Gehrels, N., Chincarini, G., Giommi, P., et al., ApJ, 611, 1005

Nousek, J.A., Kouveliotou, C., Grupe, D., et al., ApJ, 642, 389

Sari, R., Piran, T., \& Narayan, R., 1998, ApJ, 497, L17

Sari, R., Piran, T., \& Halpern, J.P., 1999, ApJ, 519, L17

Schulze, S., Klose, S., Björnsson, G., et al., 2011, A\&A, 526, A23 
